\title{
Wahn und Positivsymptome stark reduziert
}

Fragestellung: Untersuchung der Akzeptanz und Wirksamkeit des metakognitiven Trainings (MKT) bei Schizophrenie.

Hintergrund: MKT vereint Elemente von Psychoedukation und kognitiver Verhaltenstherapie und wurde für Patienten mit Schizophrenie entwickelt. Viele Studien zeigten, dass Wahn durch die Neigung zu kognitiven Verzerrungen beim Sammeln, Bewerten und Verarbeiten von Informationen begünstigt wird. Beispiele hierfür sind das Ziehen voreiliger Schlüsse, das Festhalten an einer anfänglich bezogenen Position sowie die erhöhte Sicherheit hinsichtlich falscher Überzeugungen. Das MKT zielt darauf ab, die kognitiven Verzerrungen, die mit Wahnvorstellungen und Problemen in der Perspektivübernahme sowie in der sozialen Wahrnehmung assoziiert sind, zu verringern, bevor die Hauptsymptome der Schizophrenie bearbeitet werden. Zudem versucht das MKT, den im Vergleich zu gesunden Kontrollen verringerten Selbstwert der Patienten mit Schizophrenie zu stärken. Dabei verbinden alle acht Module des Gruppentrainings theoretische Wissensvermittlung mit praktischen Übungsaufgaben. Außerdem gibt es eine ebenfalls strukturierte Form des MKT für die Einzeltherapie (MKT+) mit Übungen, die an die individuellen Bedürfnisse und Probleme des Patienten angepasst sind.

Eichner C, Berna F. Acceptance and efficacy of metacognitive Training (MCT) on positive symptoms and delusions in patients with schizophrenia: a meta-analysis taking into account important moderators. Schizophr Bull 2016; Jan 8. pii: sbv225. [Epub ahead of print]
Patienten und Methodik: In dieser Metaanalyse wurden 15 Studien berücksichtigt, die die Effekte des MKT bei Patienten mit einer Diagnose aus dem schizophrenen Formenkreis (nach DSM-IV-TR-Kriterien) auf die Positivsymptomatik allgemein, Wahn im speziellen und/oder die Akzeptanz des MKT als Intervention verglichen mit einer Kontrollgruppe untersuchten. Fünf Studien untersuchten dabei nur ein Konstrukt, vier Studien zwei Outcome-Variablen und sechs Studien erhoben Positivsymptomatik, Wahn und Akzeptanz des MKT. Es wurden sowohl Studien mit individualisierter MKT+ als auch Arbeiten mit dem MKT-Gruppenprogramm eingeschlossen. Moderationseffekte wie RaterVerblindung, Randomisierung, unvollständige Outcome-Daten und hohe Drop-out-Raten, aktive Kontrollgruppe und Publikationsbias wurden ebenso untersucht wie der Unterschied zwischen dem individuellen und dem Gruppen-MKT-Training.

Ausgewertet wurden die Daten von 408 MKT- und 399 Kontrollpatienten. Die Positivsymptomatik wurde mithilfe der Subskala der Positive and Negative Syndrome Scale (PANSS) gemessen, die Wahnausprägung durch die Items der Psychotic Symptom Rating Scales (PSYRATS) oder der Delusion Inventory von Peter et al. (PDI-21) und die subjektive Akzeptanz der Intervention mit einem Akzeptanzfragebogen erfasst.

Ergebnisse: Durchweg zeigten sich signifikante Effekte für die Reduktion von Positivsymptomen und Wahnvorstellungen in der MKT-Bedingung, wobei sich diese Effekte im kleinen bis mittleren Bereich bewegten. Ein großer Effekt zeigte sich bei der Akzeptanz der Intervention, die beim MKT signifikant höher ausfiel als bei den Kontrollinterventionen. Bei Berücksichtigung der potenziellen Verzerrungen verringerte sich der Effekt von Wahn und Positivsymptomen, blieb jedoch weiterhin signifikant. Die Effekte des individuellen Trainings waren höher als beim Gruppentraining, der Unterschied war nicht signifikant.

Schlussfolgerungen: MKT ist bei Schizophrenie sicher und effektiv und kann im klinischen Einsatz empfohlen werden.

\section{- Kommentar von Kathrin Gählert und Nadine Dreimüller, Mainz}

\section{Neue Therapieform für Schizophrenie}

Diese Arbeit liefert eine gute Zusammenfassung der Wirksamkeit des MKT und kann als Anlass genommen werden, das MKT sowohl im Gruppensetting als auch in der individuellen Einzeltherapie vermehrt zu nutzen. Das psychologische Training stellt eine sinnvolle und effektive Ergänzung zur pharmakologischen Behandlung von Schizophrenie dar und kann vor allem im Gruppensetting gut im Klinikalltag durchgeführt werden. Da einige vor allem akut schizophrene Patienten aus klinischer Erfahrung nicht gruppenfähig sind, ist auch der Einsatz des individualisierten Trainings ratsam. Da in der aktuellen Metaanalyse lediglich wenige Studien zum MKT+ einflossen und diese methodische Mängel wie keine Randomisierung und Verblindung der Rater aufwiesen, sind weitere qualitativ hochwertige Studien zur Überprüfung der Wirksamkeit unabdingbar. In diesem Rahmen wäre auch die differenziertere Betrachtung von der unterschiedlich schweren Ausprägung der
Krankheit und deren Zusammenhang mit der Wirksamkeit des Gruppen- und Einzeltrainings ratsam. Außerdem wäre es wichtig, auch Langzeiteffekte und die Auswirkung des Trainings auch in Bereichen des sozialen Lebens zu erfassen.

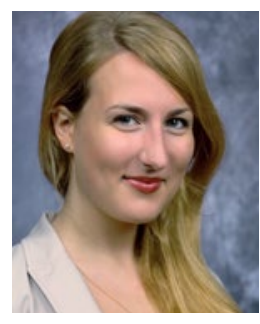

Dipl.-Psych. Kathrin Gählert, Mainz

Klinik für Psychiatrie und Psychotherapie Universitätsmedizin der Johannes GutenbergUniversität Mainz

E-Mail: kathrin.gaehlert@unimedizin-mainz.de 